\title{
Enhanced Dragonfly Algorithm based K-Medoid Clustering Model for VANET
}

\section{Dr. Joy Iong-Zong Chen,}

Professor,

Department of Communication Engineering,

Da-Yeh University, 112 Shan -Jeau, Rd.Da-Tsuen, Chang-Hua, 51505,

Taiwan R.O.C

\section{P. Hengjinda,}

Department of Industrial Robotics and Automation System,

Phetchaburi Rajabhat University,

Phetchaburi,

Thailand.

\begin{abstract}
A VANET or vehicular Ad Hoc Network is known for its fast topology transition and node mobility, contributing to its attributes as an ad hoc network. The aspect of gathering the nodes, making this system extremely vigorous is known as clustering. However, in certain cases, it is not possible to keep track of the nodes which will results in network issues due to energy insufficiency during execution. Hence this will lead to primary energy management problems faced during the routing protocol which take into consideration the node lifetime. To address this discrepancy, we have proposed a novel optimization technique based on clustering. It has been observed that the proposed methodology will further improve the effectiveness of $\mathrm{V} 2 \mathrm{~V}$ communication. In this paper, clustering of the vehicle nodes is done using K-Medoid clustering model and are then used to improve energy efficiency. A metaheuristic algorithm is used to establish an energy efficient communication methodology. Based on the simulation analysis performed, it is seen that this methodology requires lesser execution time and improves the nodes' energy efficiency.
\end{abstract}

Keywords: VAENT; EDA algorithm; energy efficient routing protocol; V2V communication, metaheuristic algorithm 
Journal of ISMAC (2021)

Vol.03/ No.01

Pages: 50-59

http://irojournals.com/iroismac/

DOI: https://doi.org/10.36548/jismac.2021.1.005

\section{Introduction}

In today's scenario, roads are seen to be large frameworks that comprise of multiple resources such as vehicles, sensors, computers, road-side foundation etc. The VANETs provide the apt platform for trading information between the roadside components and the clients without the need for network supplier. A number of researchers have researched the various network problems dealt with VRC, V2V and V1I technology with respect to the role of Intelligent Transportation systems. As the use of green communication is gaining popularity in the recent years, it has led to the use of such energy utilization in remote communications. It uses the sending probability and dimension of transmission power. In every round, the consumed energy is picked up by the node and is used for sending traffic information. Based on the convictions, the nodes are also refreshed in a periodic manner. Similarly in a large-scale Wireless Sensor Network (WSN), the many dead nodes are decreased along with an increase in energy efficiency using other protocols [1]. The aim of this algorithm [2] is to identify the base inactivity and efficiency of energy usage in a given lossy network. The major aim of such energy saving protocols is to decrease the utilization of energy in the WSN and a subsequent increase in operational lifetime by means of the shortest routing path. Similarly, energy utilization can also be adjusted based on the partitioning of the network when using energy balancing protocol [3]. Hence VANET will prove to be dynamic network that incorporates a variable and outsized network in order to enhance the communication with vehicles. Based on the movement of bearing and the areas of destination node and the source node, the optimal path between optimization and clustering model is chosen to transmit information between the source node as well as the destination node analysing the utilization of moving vehicles as a means of versatile transfer of data. Thus a number of VANET ventures have been incorporated and introduced by various academic establishments, businesses and governments, globally for over a decade now.

\section{Related Works}

As the demand for download of data is increasing in control utilization and vehicular clients, there is also a subsequent increase in the roadside unit and vehicular aspects. In [4] an energy efficient system based on multicast, operating using scheduling algorithm using artful information is examined. Here the optimum clients' counts as well as the optimum data rate 
are examined. Hence there is no need for knowledge on channel data state during the transmission process. Versatility and dynamic topology are the most crucial aspects of a VANET and the vehicles will be going in several directions at varying speeds. Since the WSNs execute their output based on the lifetime of the nodes, the constrained energy assets of the nodes are tested and examined by the authors in [5]. A number of Quality of Service (QoS) parameters are examined to determine the various difficulties and ways in which they can be tacked using efficient distributed routing protocol that is based on its position along with estimation of data transmission along with spotlights. A subclass of MANET is called the VANET, even though it is known to have a range of vehicles with high versatility with disposition to deliver to transmit successfully, the variation in network topology such as different node thickness of the vehicles and change in the road choice as observed by the authors in [6]. Characteristics such as the total number of clusters created and network overhead value are used to identify a particular vehicle from the other.

Researchers in [7] have insisted on discussions between cars in a remote manner using VANETs where attackers might affect genuineness, security and secrecy of the information that could affect the insurance. A VANET framework is built taking into consideration the various existing threads and safety challenges. Using information sent from a vehicular test bed, the reliability of many applications were examined using good portability information. When using Fog computing, it was possible to properly estimate the timings of bus as well as traffic peculiarities by interfacing with the cloud. In [8] the authors have improved the steering execution with respect to higher availability and better transmission time. VANET can be improved using FF algorithm which can further improve its performance using transfer of packets between the goal vehicle and the source vehicle. In [9] the security challenges and protection mechanisms were laid out to ensure the stability of a network. This work deals with recent security issues faced while transferring information with respect to cryptographic transmission in VANETs [10].

\section{Proposed Work}

In this paper we have incorporated an energy efficient routing protocol that is used to solve the issue of optimization in the vehicular communication framework. Here the V2V communication model or VIR communication model is used along with the vehicle network 
topology. First the cluster head as is managed with the help of clustering algorithm which is followed by the implementation of an energy efficient algorithm. Due to the increase in population of developing nations, the subsequent increase in the traffic is of high importance. Accordingly, the security of the road is to be concentrated in order to make movement of vehicles as efficient as possible amidst road traffic [11-12]. This is where the energy efficient algorithm and clustering methodologies are used to ensure that communication between and among vehicles take place in a well-coordinated and streamlined manner. When there is such a vast source of ways to broadcast information with limited means of message conveyance, it will result in blockage of information while communicating them resulting in broadened delay, low energy efficiency and packet dropping. Hence we propose a novel methodology that ensures optimal information transmission with an energy-efficient routing protocol that takes into consideration the energy parameter with optimal routing technique.

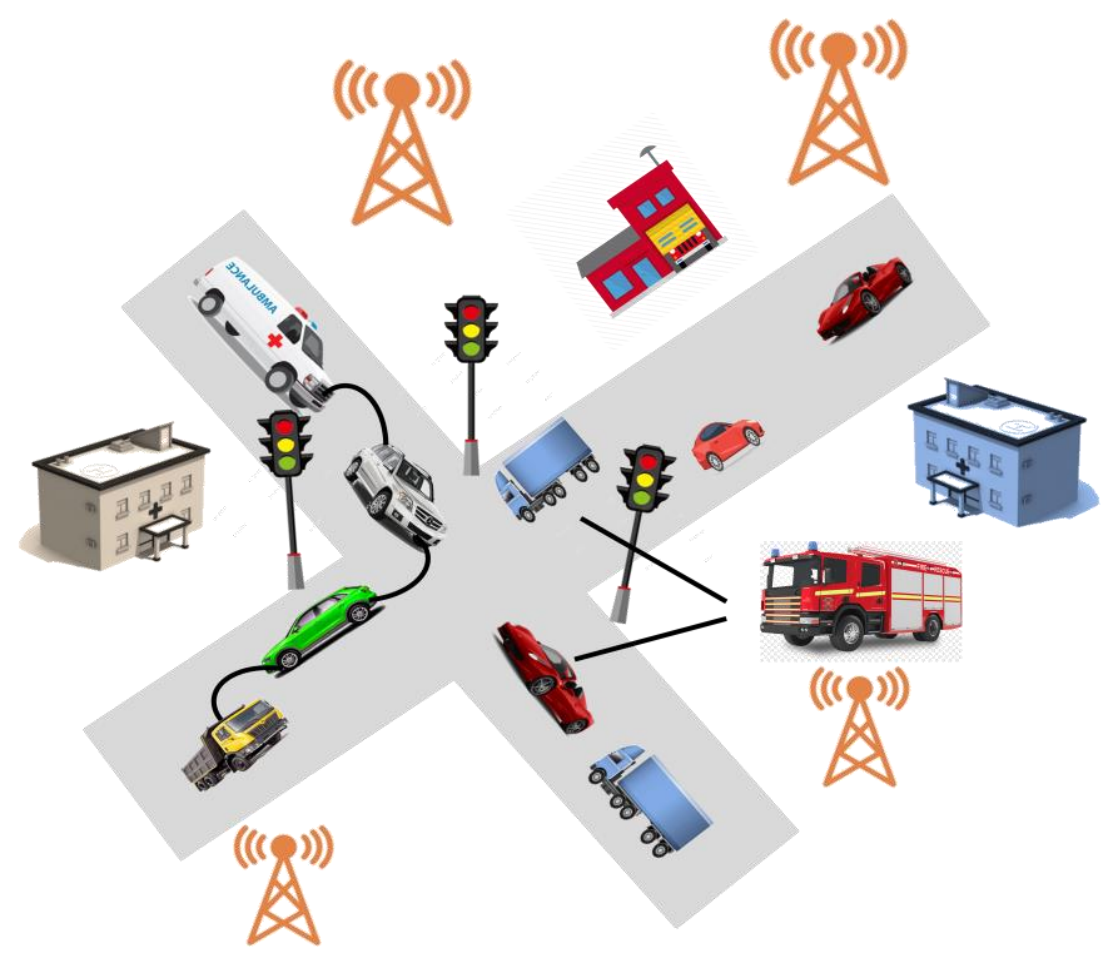

Fig.1. Basic Network Topology for V2V Communication

Fig.1. represents the network topology of the proposed work along with vehicular development and a subset of the city map with a view of the streets and the various traffic congestions. In this paper, we have made use of 200 vehicular nodes where every node 
transmits information with each other without a GPS. Some reference nodes have also been incorporated with a GPS [13] to have an accurate knowledge on the data position. Here the two sides of the road representing the destination and the source node are segregated into two sections by means of a plane. The VANET clustering system is used in order to build the clusters such that they will not play in contrast to that of their arrangement. In this work we have incorporated K-medoid clustering methodology that uses nodes from 200 vehicles in order to form a complete cluster. If a node form the cluster is rejected due to some reason, it will join the neighbouring group of nodes. If there is much difference between the node with which they will be joining, the can join other groups.

With K-Medoid clustering, the information things ' $\mathrm{k}$ ' are chosen within a cluster. Within a particular cluster, the various particulars essential are incorporated with respect to the nodes that fall within its medoids. Based on its properties, the node will either fall under one medoid or another that is closest. The different stages involved in K-medoid clustering are:

- Initializing: Medoids are chosen as $\mathrm{K}$ value depending on the different points of data nodes.

- Choosing the Medoids: Based on the distance between the data points of two objects, the value of medoid is selected. Accordingly, the distance can be evaluated using the formula:

$$
D=\sum_{j=1}^{n}\left(F_{j}-S_{j}\right)^{2}
$$

- Cluster Generation: Each cluster is allocated to the medoid value that is closest to the object present.

- Medoid Updation: The purpose of evaluating the new medoids in every cluster is to ensure that the distance between the cluster and the object is minimal.

- Nodes in Medoid: Clustering is achieved by allocating every object that is closer to the medoid. The separation from the objects will be used to determine the distance between the medoids and the objects. On incorporating the K-medoid algorithm, the distance measured is used to analyse the gathering of the various nodes. The clustering process is done between the vehicles using V2V as shown in the Fig.2. 


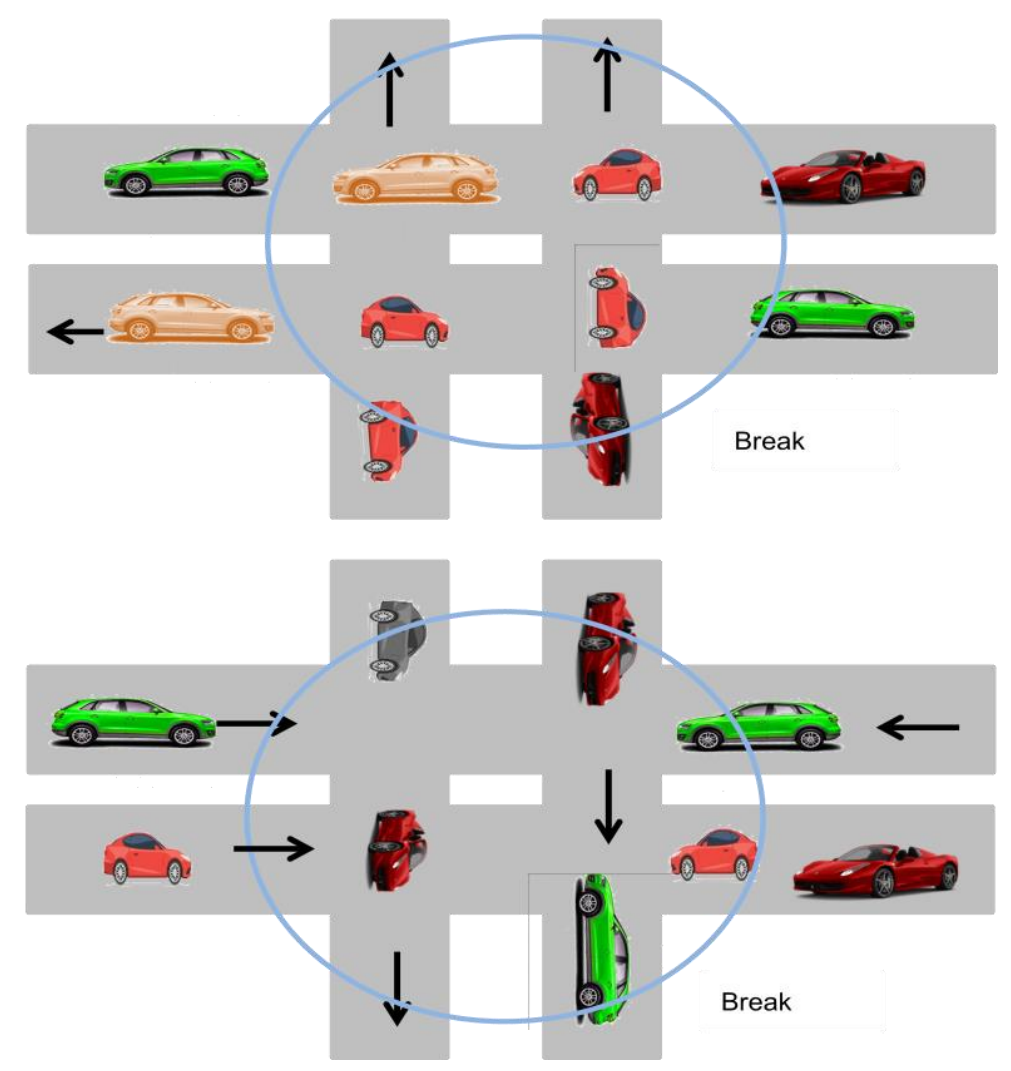

Fig.2. Clustering of vehicle nodes (200 nodes considered)

Portability measurements such as acceleration, position and speed are used to determine the cluster head formation for the vehicle nodes. The cluster head is chosen depending on the smallest average distance of the vehicle with respect to a cluster.

- Closest Velocity to Average: Based on the difference between the average velocity and the candidate's velocity, the cluster head is chosen with respect to the vehicle proximity.

- Closest Position to Average: Based on the difference between the average position and the candidate's position, the cluster head is chosen with respect to the vehicle proximity.

The major goal is to develop an efficient routing schedule for $\mathrm{V} 2 \mathrm{~V}$ communication, taking into consideration the network parameter optimization. By decreasing the use of energy by the vehicular nodes, the fitness parameters are determined. The target work is denoted as shown below:

$$
O F(V 2 V)=\{\min (E C)\}
$$


Optimization techniques such as enhanced dragonfly algorithm are used to decrease the value of energy constraint. The dragonfly algorithm is based on the characteristics of the dragon flies which tend to chase away smaller insects, behaving like predators. The dynamic and static swarming particles form the basis of the DA algorithm. These two swarming practices use exploitation and exploration of meta heuristics as their tool for optimization. Similarly, the enhanced function does not take into consideration the neighbourhood ideal and will be used to decrease the probability of catching. The dragonflies or vehicle nodes can be initialized using the formula:

$$
D_{i}=D_{1}, D_{2}, D_{3}, \ldots \ldots \ldots, D_{n} \text { such that } \mathrm{i}=1,2,3 \ldots \mathrm{n}
$$

There are five stages in which the behaviour of dragonflies can be analyzed such as attraction, cohesion, alignment, distraction, attraction and separation.

\section{Results and Discussions}

The performance of the proposed algorithm is examined using NS2 software. The analysis is made based on the network parameters such as throughput, network lifetime, packet delivery ratio (PDR) and energy consumption. Based on the optimization procedure and the clustering algorithm, the throughput analysis is performed as shown in Fig. 3. The proposed enhanced dragonfly algorithm shows better results when compared with its predecessors when measurements of throughput are taken in intervals of 50. Similarly, Fig.4 shows the PDR for varying vehicle quantity and it is observed that the delivery ratio is high as the number of vehicles increases.

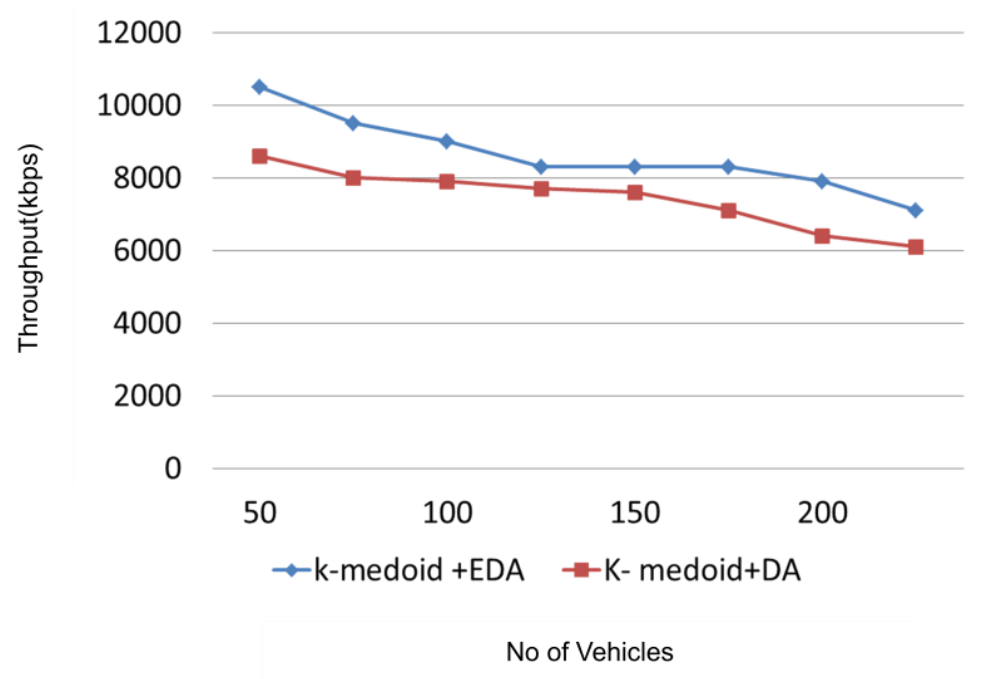

Fig.3 Throughput Analysis using K-Medoid EDA algorithm 
Vol.03/ No.01

Pages: 50-59

http://irojournals.com/iroismac/

DOI: https://doi.org/10.36548/jismac.2021.1.005

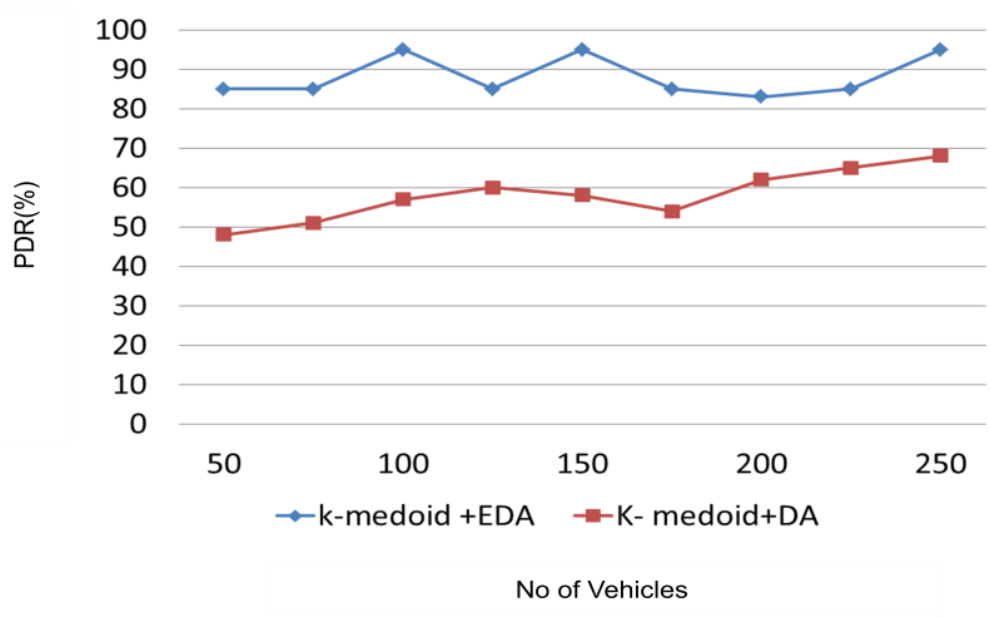

Fig.4 PDR Analysis using K-Medoid EDA algorithm

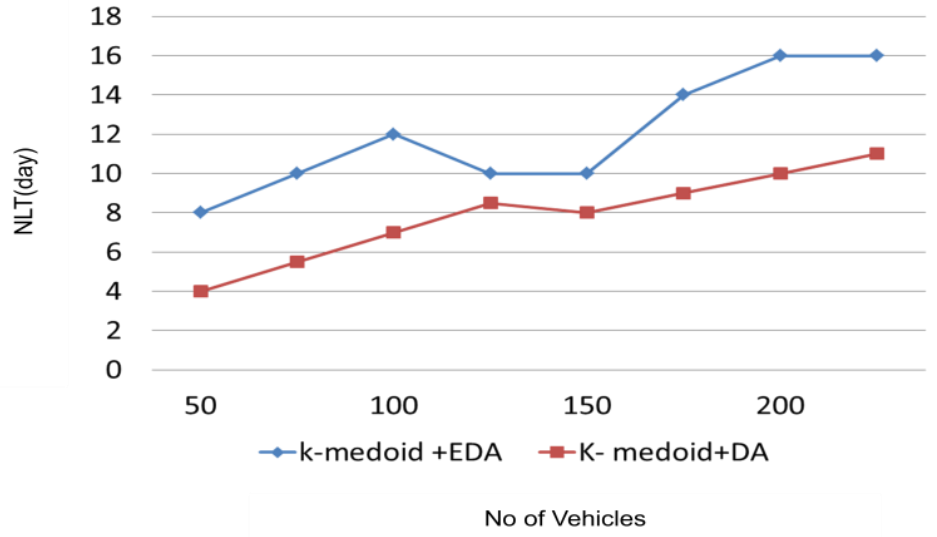

Fig.5 NLT Analysis using K-Medoid EDA algorithm

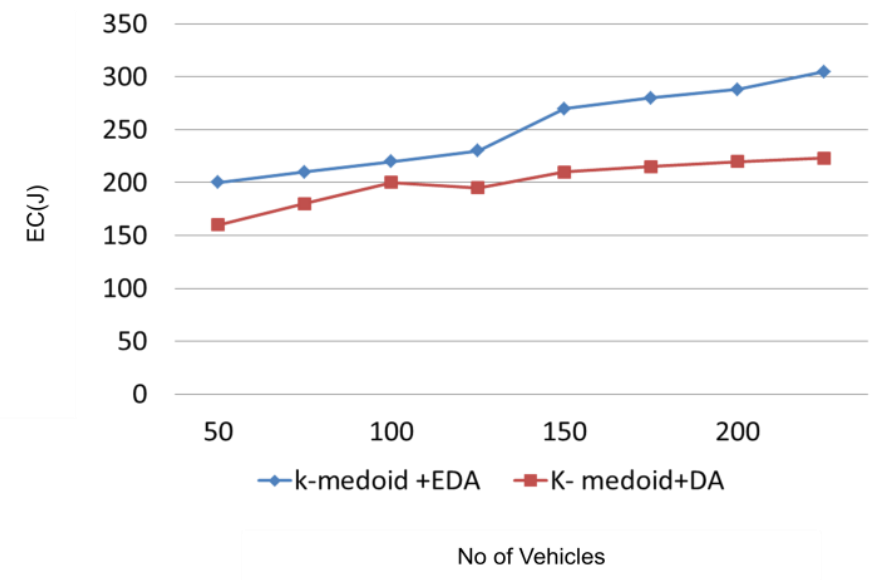

Fig.6 EC Analysis using K-Medoid EDA algorithm

ISSN: 2582-1369 (online)

Submitted:20.01.2021

Revised: 28.02.2021

Accepted: 22.03.2021

Published: 07.04.2021 
Journal of ISMAC (2021)

Vol.03/ No.01

Pages: 50-59

http://irojournals.com/iroismac/

DOI: https://doi.org/10.36548/jismac.2021.1.005

Fig.5 and Fig.6 summerise the impact of the proposed algorithm in analysis of NLT and EC respectively. It is observed that when compared with existing algorithm, the enhanced dragon fly model shows lesser amount of energy spent and improved NLT ratio.

\section{Conclusion}

In this paper, we have introduced a K-Medoid clustering using Enhanced Dragonfly algorithm for identifying and communicating with the nodes present in the cluster, taking into consideration the energy utilization factor. The vehicle nodes are clustered together using a cluster head at every round which can transmit the message between the base station and the nodes. With the aid of EDA algorithm, the most energy efficient route to transmit information between the clusters using V2V communication is determined. The experimental analysis and simulation results indicate that the proposed enhanced dragonfly algorithm performs better in terms of throughput, EC analysis, PDR and NLT output. This work can further be expanded to a broader scope to increase the QoS and also address the issues of cost consideration.

\section{References}

[1] Shilaja, C., \& Arunprasath, T. (2019). Internet of medical things-load optimization of power flow based on hybrid enhanced grey wolf optimization and dragonfly algorithm. Future Generation Computer Systems, 98, 319-330.

[2] Wang, Y., Wang, M., Shen, L., Sun, X., Shi, G., Ma, W., \& Yan, X. (2018). Highperformance flexible surface-enhanced Raman scattering substrates fabricated by depositing Ag nanoislands on the dragonfly wing. Applied Surface Science, 436, 391397.

[3] Rahman, C. M., \& Rashid, T. A. (2019). Dragonfly algorithm and its applications in applied science survey. Computational Intelligence and Neuroscience, 2019.

[4] Stevani, C. V., de Faria, D. L., Porto, J. S., Trindade, D. J., \& Bechara, E. J. (2000). Mechanism of automotive clearcoat damage by dragonfly eggs investigated by surface enhanced Raman scattering. Polymer Degradation and Stability, 68(1), 61-66.

[5] Kadoya, T., Suda, S. I., \& Washitani, I. (2004). Dragonfly species richness on manmade ponds: effects of pond size and pond age on newly established assemblages. Ecological Research, 19(5), 461-467. 
[6] Karunakaran, V. (2019). a stochastic development of cloud computing based task scheduling ALGORITHM. Journal of Soft Computing Paradigm (JSCP), 1(01), 41-48.

[7] Hu, Z., \& Deng, X. Y. (2014). Aerodynamic interaction between forewing and hindwing of a hovering dragonfly. Acta Mechanica Sinica, 30(6), 787-799.

[8] Halberstadt, A. L., Chatha, M., Stratford, A., Grill, M., \& Brandt, S. D. (2019). Comparison of the behavioral responses induced by phenylalkylamine hallucinogens and their tetrahydrobenzodifuran ("FLY") and benzodifuran ("DragonFLY") analogs. Neuropharmacology, 144, 368-376.

[9] Yıldız, B. S., \& Yıldız, A. R. (2019). The Harris hawks optimization algorithm, salp swarm algorithm, grasshopper optimization algorithm and dragonfly algorithm for structural design optimization of vehicle components. Materials Testing, 61(8), 744748.

[10] Sureshkumar, K., \& Ponnusamy, V. (2019). Power flow management in micro grid through renewable energy sources using a hybrid modified dragonfly algorithm with bat search algorithm. Energy, 181, 1166-1178.

[11] Manoharan, S. (2019). A smart image processing algorithm for text recognition information extraction and vocalization for the visually challenged. Journal of Innovative Image Processing (JIIP), 1(01), 31-38.

[12] Rahimunnisa, K. (2019). Hybrdized genetic-simulated annealing algorithm for performance optimization in wireless adhoc network. Journal of Soft Computing Paradigm (JSCP), 1(01), 1-13.

[13] Senthilkumar, M., Kavitha, V. R., Kumar, M. S., Raj, P. A. C., \& Shirley, D. R. A. (2021, March). Routing in a Wireless Sensor Network using a Hybrid Algorithm to Improve the Lifetime of the Nodes. In IOP Conference Series: Materials Science and Engineering (Vol. 1084, No. 1, p. 012051). IOP Publishing. 